\title{
Apelin-13 induces autophagy in hepatoma HepG2 cells through ERK1/2 signaling pathway-dependent upregulation of Beclin1
}

\author{
QIULIN HUANG ${ }^{1}$, XUAN LIU $^{1}$, CHAO CAO ${ }^{1}$, JUNYUE LEI ${ }^{1}$, DONG HAN ${ }^{1}$, \\ GUODONG CHEN ${ }^{1}$, JIA YU ${ }^{1}$, LINXI CHEN ${ }^{2}$, DEGUAN LV ${ }^{1}$ and ZHONGYU LI ${ }^{3}$ \\ ${ }^{1}$ Department of General Surgery, First Affiliated Hospital of University of South China; \\ ${ }^{2}$ Institute of Pharmacy and Pharmacology; ${ }^{3}$ Department of Microbiology, School of Medicine, \\ University of South China, Hengyang, Hunan 421001, P.R. China
}

Received August 26, 2014; Accepted June 11, 2015

DOI: $10.3892 / 01.2015 .3991$

\begin{abstract}
The aim of the present study was to investigate the effect of Apelin-13 on autophagy in hepatocellular carcinoma HepG2 cells and the underlying mechanism of the effect. The HepG2 cells were treated with Apelin-13 at a final concentration of $0.0001,0.001,0.01$ and $0.1 \mu \mathrm{mol} / 1$ for $24 \mathrm{~h}$. Cells were also treated with $10 \mu \mathrm{mol} / 1 \mathrm{PD} 98059$ for $24 \mathrm{~h}$. The expression of the extracellular signal-regulated kinase (ERK)1/2, phosphorylated ERK1/2 (pERK1/2) and Beclin1 proteins were detected by western blot analysis. Beclin1 mRNA expression was also detected by reverse transcription-polymerase chain reaction. Autophagy was observed using fluorescence microscopy subsequent to monodansylcadaverine (MDC) staining. Following treatment with the various concentrations of Apelin-13, the expression of the ERK1/2 protein remained at a similar level, whereas the expression of pERK $1 / 2$ increased in a dose-dependent manner. Compared with the control group, the increase was significant $(\mathrm{P}<0.05)$. Similarly, Beclin1 expression was upregulated at the protein and mRNA levels by Apelin-13 treatment in a dose-dependent manner and was significantly increased compared with the control group. However, following treatment with the Apelin-13 inhibitor PD98059, the expression of pERK1/2, Beclin1 protein and Beclin1 mRNA were significantly decreased $(\mathrm{P}<0.05)$. In addition, Apelin-13 induced the autophagy of HepG2 cells in a dose-dependent manner, as revealed by MDC staining. PD98059 inhibited autophagy of HepG2 cells induced by Apelin-13. Therefore, Apelin-13 may
\end{abstract}

Correspondence to: Dr Qiulin Huang, Department of General Surgery, First Affiliated Hospital of University of South China, 69 Chuanshan Road, Hengyang, Hunan 421001, P.R. China E-mail: hq1107@sina.com

Dr Zhongyu Li, Department of Microbiology, School of Medicine, University of South China, 28 Changsheng Road, Hengyang, Hunan 421001, P.R. China

E-mail: 1zhy7404@sina.com

Key words: extracellular signal-regulated kinase 1/2, expression levels, phosphorylation promote autophagy in HepG2 cells by inducing the phosphorylation of ERK1/2 and upregulating the expression of Beclin1.

\section{Introduction}

In previous years, the association between tumor development and autophagy has received increasing attention. As the first gene identified to inhibit tumorigenesis through lysosome degradation, Beclin1 has been widely studied. The mammalian Beclin1 gene encodes a B-cell lymphoma 2-interacting coiled-coil protein that possesses structural similarities to the yeast autophagy protein. The human Beclin1 gene, which contains 12 exons, is located on human chromosome 17q21. It has previously been reported that Beclin1 is a mammalian autophagy gene that inhibits tumorigenesis (1-3). Beclin1, as a key factor that mediates the localization of other autophagy proteins into the precursor of autophagic bodies, is involved in the regulation of mammalian autophagy formation (4).

Extracellular signal-regulated kinase (ERK) $1 / 2$ is a protein involved in the Ras/Raf-1/ERK1/2 signaling pathway that is important for autophagy (5). Sodium arsenite induces autophagy through ERK1/2 phosphorylation in human uroepithelial cells (6). Apelin is isolated from an endogenous ligand from bovine gastric secretion (7). Apelin-13 is one of the most active protein fragments in the Apelin/Apelin receptor system, and promotes the phosphorylation of ERK1/2 in rat vascular smooth muscle cells in a concentration-dependent manner (8).

In the present study, the effect of Apelin-13 on the protein and mRNA expression of ERK1/2, phosphorylated ERK (pERK)1/2 and Beclin1 was determined. The effect of Apelin-13 on autophagy in HepG2 cells was also investigated.

\section{Materials and methods}

Reagents and instruments. RPMI-1640, fetal bovine serum (FBS), trypsin and dimethyl sulfoxide (DMSO) were purchased from Beyotime Institute of Biotechnology (Hangzhou, Zhejiang, China). Apelin-13 and PD98059 were purchased from Santa Cruz Biotechnology, Inc. (Dallas, TX, USA). Monodansylcadaverine (MDC) and mouse monoclonal $\beta$-tubulin (D-10; sc-5274), rabbit polyclonal pERK (Thr 177/Thr 160; sc-23759-R), mouse monoclonal ERK (C-9; 
sc-514302) and mouse monoclonal Beclin-1 (G-11; sc-48381) antibodies were purchased from Santa Cruz Biotechnology, Inc. Horseradish peroxidase-conjugated rabbit anti-mouse and mouse anti-rabbit secondary antibodies, and the hyper-pure RNA, PrimeScript reverse transcription-polymerase chain reaction (RT-PCR), enhanced chemiluminescence western blot and bicinchoninic acid (BCA) protein assay kits were purchased from Beijing ComWin Biotech Co., Ltd.(Beijing, China). The primers for RT-PCR were synthesized by Sangon Biotech Co., Ltd. (Shanghai, China).

An IX73 inverted and reflected fluorescent microscope was purchased from Olympus Optical Co., Ltd. (Tokyo, Japan). The FlourChem E HD2 and AlphaImager 2200 gel imaging systems were purchased from ProteinSimple (San Jose, CA, USA).

Cell lines and culture. Hepatocarcinoma HepG2 cells were provided by the Tumor Research Institute of University of South China. The cells were cultured in RPMI-1640 medium supplemented with $10 \% \mathrm{FBS}$, and maintained in a humidified incubator at $37^{\circ} \mathrm{C}$ and in a $5 \% \mathrm{CO}_{2}$ atmosphere. Adherent cells were trypsinized using $0.25 \%$ trypsin-EDTA, harvested and re-plated into flasks every 2-3 days. Cells in the logarithmic phase of growth were used in the subsequent analyses.

Experimental groups and treatments. The cells were treated with Apelin-13 and PD98059. Apelin-13 was dissolved in phosphate-buffered saline (PBS) and PD98059 was dissolved in DMSO. In the Apelin-13 group, the cells were treated with Apelin-13 at final concentrations of 0.0001, 0.001, 0.01 and $0.1 \mu \mathrm{mol} / 1$ for $24 \mathrm{~h}$. In the PD98059 group, the cells were treated with $10 \mu \mathrm{mol} / \mathrm{l} \mathrm{PD} 98059$ for $24 \mathrm{~h}$. In the Apelin-13 and PD98059 groups, the cells were treated with $0.1 \mu \mathrm{mol} / 1$ Apelin-13 and $10 \mu \mathrm{mol} / 1$ PD98059 for $24 \mathrm{~h}$, respectively. The cells treated with $10 \%$ FBS and DMSO were used as the control groups.

Western blot analysis. The samples were lysed in $100 \mu \mathrm{l}$ of lysis buffer (RIPA:PMSF ratio, 94:6). Subsequent to incubating the samples on ice for $30 \mathrm{~min}$, the cell lysate was centrifuged at $18,000 \mathrm{xg}$ for $8 \mathrm{~min}$ at $4^{\circ} \mathrm{C}$ and the supernatant was collected. The protein concentration in the supernatant was quantified using the BCA kit. The proteins were then separated by SDS-PAGE and transferred onto a nitrocellulose membrane. Subsequent to blocking, the membrane was incubated at $21^{\circ} \mathrm{C}$ overnight with mouse anti- $\beta$-tubulin (dilution, 1:250), mouse anti-ERK1/2 (dilution, 1:250), rabbit anti-pERK1/2 (dilution, 1:250) and mouse anti-Beclin1 (dilution, 1:250) monoclonal primary antibodies. Subsequent to washing, the mouse anit-rabbit and rabbit anti-mouse secondary antibodies were added at dilutions of 1:10,000 and 1:5,000, respectively, and the membrane was incubated at $21^{\circ} \mathrm{C}$ for $4 \mathrm{~h}$. Finally, the membrane was developed using enhanced chemiluminescence reagent (Beyotime Institute of Biotechnology). The developed film was scanned using the AlphaImager 2200 gel imaging system. The western blot images were analyzed using GraphPad Prism 5, which was purchased from GraphPad Software, Inc., (La Jolla, CA, USA). $\beta$-tubulin was used as an internal control. The relative expression of ERK1/2, pERK1/2 and Beclin1 was calculated based on the grayscale value of $\beta$-tubulin.
RT-PCR assay. Total RNA was extracted from HepG2 cells, and the expression of Beclin1 mRNA was detected using the PrimeScript RT-PCR kit. According to the manufacturer's instructions, the purity and concentration of the RNA was calculated using a DNA/RNA calculator (Pharmacia, Fairfield, CT, USA). For the amplification of Beclin1, PCR was performed with pre-denaturation step at $95^{\circ} \mathrm{C}$ for $5 \mathrm{~min}$, 35 cycles consisting of denaturation at $94^{\circ} \mathrm{C}$ for $30 \mathrm{sec}$, annealing at $58^{\circ} \mathrm{C}$ for $40 \mathrm{sec}$ and extension at $72^{\circ} \mathrm{C}$ for $1 \mathrm{~min}$, and a final extension at $72^{\circ} \mathrm{C}$ for $10 \mathrm{~min}$. GAPDH was used as the internal reference. The PCR products were separated by electrophoresis under a constant voltage of $80 \mathrm{~V}$ in a $12 \mathrm{~g} / \mathrm{l}$ agarose gel for $30 \mathrm{~min}$. The gel was then observed and images of the gel were captured using the AlphaImager 2200 gel imaging analysis system. This reaction was conducted three times. The primer sequences used were as follows: GAPDH sense, 5'-ACCACAGTCCATGCCATCAC-3' and anti-sense, 5'-TCCACCACCCTGTTGCTGTA-3'; Beclin1 sense, 5'-CTA AGTCGTCCAACAACAGCAC-3' and anti-sense, 5'-CGATGT CAAAAAGGTCCC-3'. The primers were designed and synthesized by Sangon Biotech Co., Ltd. (Shanghai, China). The relative expression level of Beclin1 was determined by the grayscale ratio of Beclin1 to GAPDH.

MDC staining and autophagy detection. Based on the method described by Biederbick et al, MDC was used to stain the autophagosomes (9). Briefly, the cells were placed on six-well plates and treated with Apelin-13 and PD98059, as aforementioned. Following incubation for $24 \mathrm{~h}, 0.05 \mathrm{mmol} / 1 \mathrm{MDC}$ was added to the plates and the cells were incubated at $37^{\circ} \mathrm{C}$ for $60 \mathrm{~min}$. The cells were then fixed with $4 \%$ paraformaldehyde for $15 \mathrm{~min}$. Subsequent to rinsing twice with cold PBS, the cells were observed and photographed by inverted fluorescence microscopy.

Statistical analysis. The data were expressed as the mean \pm standard deviation and were analyzed using SPSS software, version 13 (SPSS, Inc., Chicago, IL, USA). The differences between groups were compared using one-way analysis of variance. Multiple comparison between the groups was performed using the S-N-K method The variables in the two groups were compared by Student's $t$-test. $\mathrm{P}<0.05$ was considered to indicate a statistically significant difference.

\section{Results}

Apelin-13 upregulates the expression of pERK1/2 in HepG 2 cells. To determine the effect of Apelin-13 on the expression of ERK1/2 and pERK1/2, western blot analysis was performed. The cells were treated with Apelin-13 at final concentrations of $0.0001,0.001,0.01$ and $0.1 \mu \mathrm{mol} / 1$ for $24 \mathrm{~h}$. The cells treated with $10 \%$ FBS were used as a negative control. Representative western blot results are presented in Fig. 1A and quantitative results are reported in Fig. 1B. As indicated in Fig. 1, following treatment with Apelin-13, the expression level of ERK1/2 was not evidently changed. However, the level of pERK1/2 was increased in a dose-dependent manner. The relative expression levels of pERK $1 / 2$ in cells treated with $10 \%$ FBS or $0.0001,0.001,0.01$ and $0.1 \mu \mathrm{mol} / 1$ Apelin- 13 were $0.445 \pm 0.021,0.764 \pm 0.013,0.857 \pm 0.018,0.906 \pm 0.072$ and 

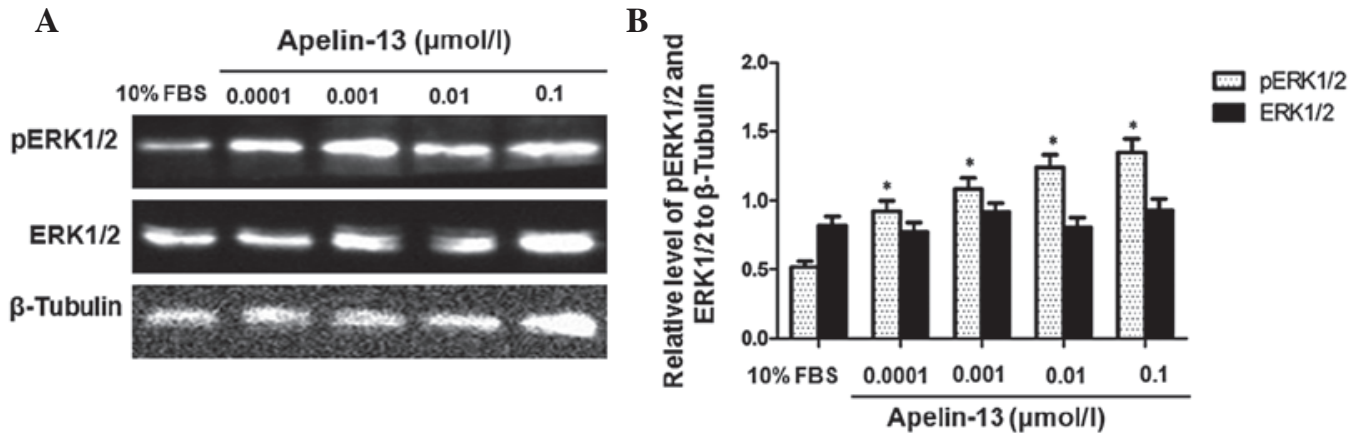

Figure 1. Analysis of the expression of ERK1/2 and pERK1/2 subsequent to treatment with Apelin-13. The cells were treated with $0.0001,0.001,0.01$ or $0.1 \mu \mathrm{mol} / 1$ Apelin-13 for $24 \mathrm{~h}$. ERK1/2 and pERK1/2 expression was then detected by western blot analysis. $\beta$-tubulin was used as an internal control. The cells treated with $10 \%$ FBS were used as negative control. (A) Representative western blotting results. (B) Quantitative western bloting results. The relative expression level of ERK1/2 and pERK1/2 was the ratio of ERK1/2 to $\beta$-tubulin and the ratio of pERK1/2 to $\beta$-tubulin, respectively. The data were presented as the mean \pm standard deviation of three independent experiments. ${ }^{*} \mathrm{P}<0.01$ vs. cells treated with $10 \%$ FBS. ERK1/2, extracellular signal-regulated kinase $1 / 2$; pERK1/2, phosphorylated ERK1/2; FBS, fetal bovine serum.

A

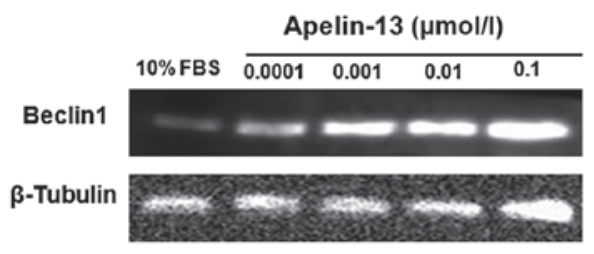

$\mathbf{C}$

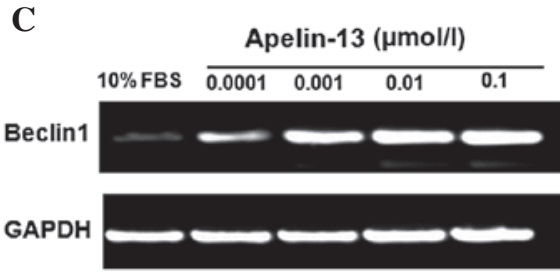

B

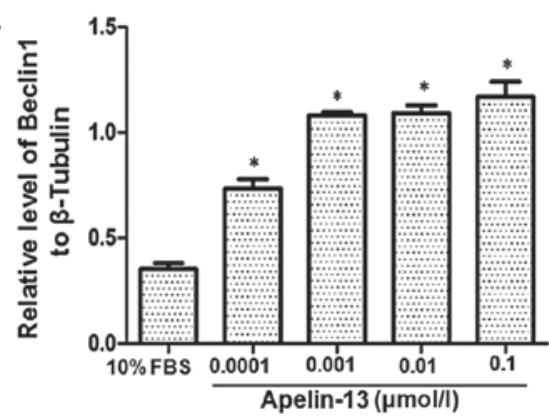

D

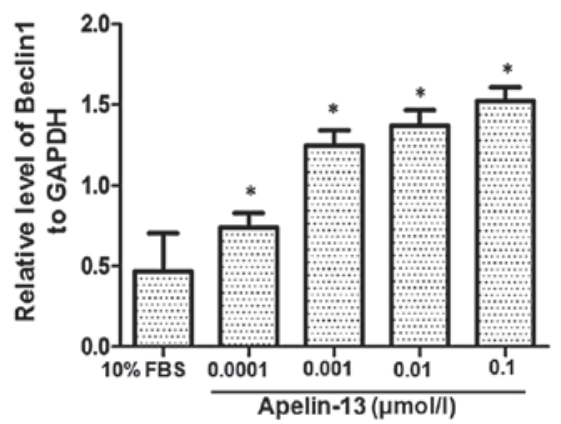

Figure 2. Analysis of the expression of Beclin1 subsequent to treatment with Apelin-13. The cells were treated with $0.0001,0.001,0.01$ or $0.1 \mu \mathrm{mol} / 1 \mathrm{Apelin}-13$ for $24 \mathrm{~h}$. The cells treated with $10 \%$ FBS were used as a negative control. Beclin1 protein expression was detected by western blot analysis. $\beta$-tubulin was used as an internal control. (A) Representative western blot results. (B) Quantitative western blot results. The relative expression level of the Beclin1 protein was the ratio of Beclin1 to $\beta$-tubulin. Beclin1 mRNA expression was analyzed by RT-PCR. GAPDH was used as an internal control. (C) Representative RT-PCR results. (D) Quantitative RT-PCR results. The relative expression level of Beclin1 mRNA was the ratio of Beclin1 to GAPDH. The data were expressed as the mean \pm standard deviation of three independent experiments. "P<0.01 vs. cells treated with $10 \%$ FBS. RT-PCR, reverse transcription-polymerase chain reaction; FBS, fetal bovine serum.

$1.019 \pm 0.041$, respectively. Following treatment with Apelin-13, the expression levels of pERK1/2 at concentrations of 0.0001 , $0.001,0.01$ and $0.1 \mu \mathrm{mol} / 1$ were significantly increased compared with the negative control group $(\mathrm{P}<0.05)$. These data indicate that Apelin-13 induced the phosphorylation of ERK1/2 and upregulated the level of pERK1/2 in HepG2 cells in a dose-dependent manner.

Apelin-13 upregulates the expression of Beclin1 protein and mRNA in HepG2 cells. To analyze the effect of Apelin-13 on the expression of Beclin1 protein, western blot analysis was performed using HepG2 cells treated with 0.0001, 0.001, 0.01 and $0.1 \mu \mathrm{mol} / 1$ Apelin-13. The negative control was performed using $10 \%$ FBS. Fig 2A presents the representative western blot results and Fig. 2B reports the quantitative western blot results. In the cells treated with $10 \%$ FBS, Beclin1 protein expression was extremely low. However, the protein expression of Beclin1 increased significantly subsequent to Apelin-13 treatment. The increase was dose-dependent. Subsequent to quantification, the relative expression of the Beclin1 protein in cells treated with $10 \%$ FBS and with 0.0001 , $0.001,0.01$ and $0.1 \mu \mathrm{mol} / 1$ Apelin-13 were $0.354 \pm 0.010$, $0.734 \pm 0.017,1.080 \pm 0.068,1.092 \pm 0.014$ and $1.168 \pm 0.024$, respectively. Compared with the cells treated with $10 \%$ FBS, the cells treated with Apelin-13 demonstrated significantly increased levels of Beclin1 expression $(\mathrm{P}<0.05)$. 

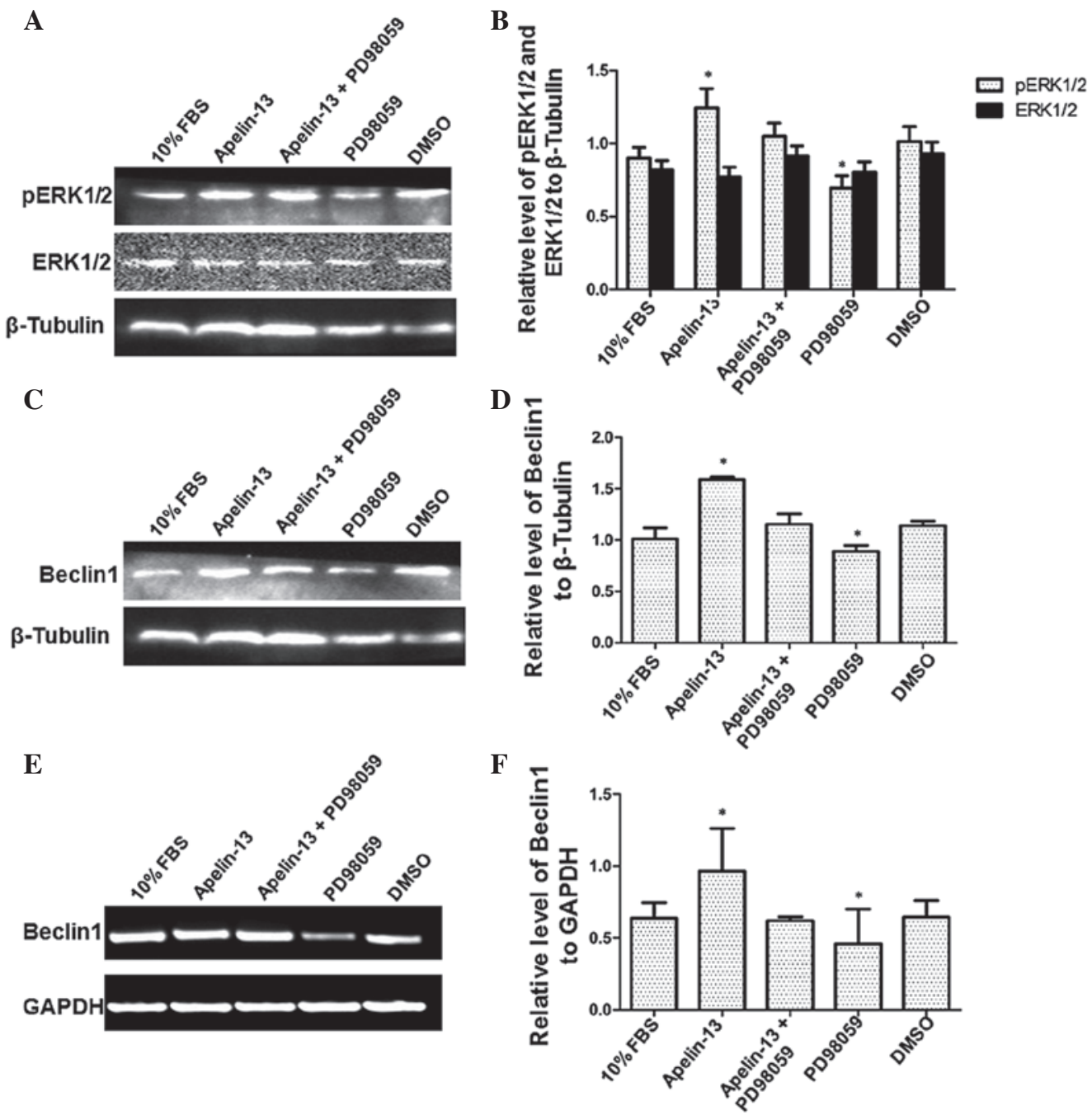

Figure 3. Analysis of ERK1/2, pERK1/2 and Beclin1 expression subsequent to treatment with Apelin-13 and PD98059. The cells were treated with $0.1 \mu$ mol/1 Apelin-13, $0.1 \mu \mathrm{mol} / 1$ Apelin-13 combined with $10 \mu \mathrm{mol} / 1$ PD98059 or $10 \mu \mathrm{mol} / 1$ PD98059 for $24 \mathrm{~h}$. The cells treated with $10 \%$ FBS and DMSO were used as negative controls. (A and B) The expression of ERK1/2 and pERK1/2 was detected by western blot analysis. (A) Representative and (B) quantitative western blot results are shown. $\beta$-tubulin was used as an internal control. The relative expression levels of ERK1/2 and pERK1/2 were calculated as the ratio of ERK1/2 to $\beta$-tubulin and the ratio of pERK1/2 to $\beta$-tubulin, respectively. (C and D) Beclin1 protein expression was detected by western blot analysis. (C) Representative and (D) quantitative western blot results are shown. $\beta$-tubulin was used as an internal control. The relative expression level of Beclin1 protein was calculated as the ratio of Beclin1 to $\beta$-tubulin. (E and F) Beclin1 mRNA expression was analyzed by RT-PCR. GAPDH was used as an internal control. (E) Representative and (F) quantitative RT-PCR results are shown. The relative expression level of Beclin1 mRNA was calculated as the ratio of Beclin1 to GAPDH. The data were expressed as the mean \pm standard deviation of three independent experiments. " $\mathrm{P}<0.01$ vs, cells treated with $10 \%$ FBS and DMSO. ERK1/2, extracellular signal-regulated kinase 1/2; pERK1/2, phosphorylated ERK1/2; FBS, fetal bovine serum; DMSO, dimethyl sulfoxide; RT-PCR, reverse transcription-polymerase chain reaction.

In addition, the effect of Apelin-13 on Beclin1 mRNA expression was also investigated by RT-PCR. GAPDH was used as an internal control. Similarly, Beclin1 mRNA was barely detectable in cells treated with $10 \%$ FBS, whereas the expression became gradually upregulated in cells treated with various concentrations of Apelin-13 (Fig. 2C and D). Quantitatively, the Beclin1 mRNA level was $0.466 \pm 0.095$ in cells treated with $10 \%$ FBS, and the Beclin1 mRNA levels were $0.737 \pm 0.038,1.246 \pm 0.038,1.370 \pm 0.039$ and $1.522 \pm 0.034$ in cells treated with $0.0001,0.001,0.01$ and $0.1 \mu \mathrm{mol} / 1$ Apelin-13, respectively. Statistically, there were significantly higher levels of Beclin1 mRNA in cells treated with Apelin-13 compared with the cells treated with $10 \%$ FBS $(\mathrm{P}<0.05)$. Overall, these results suggest that Apelin-13 stimulated the expression of Beclin1 at the protein and mRNA level in a dose-dependent manner.
Apelin-13 inhibitor PD98095 suppresses the phosphorylation of $p E R K 1 / 2$ and expression of Beclin1 induced by Apelin-13. To further verify the effect of Apelin-13 on pERK1/2 and Beclin1, the HepG2 cells were treated with the Apelin-13 inhibitor PD 98059. As aforementioned, the most notable effect of Apelin-13 was observed at the concentration of $0.1 \mu \mathrm{mol} / 1$. Therefore, $0.1 \mu \mathrm{mol} / 1$ Apelin- 13 was used in the present study. The concentration of PD 98059 used was $10 \mu \mathrm{mol} / 1$. First, western blot analysis was performed to assess the effect of Apelin-13 and PD98059 on pERK1/2 expression (Fig. 3A and B). Consistently, cells treated with Apelin-13 alone demonstrated increased levels of $\mathrm{pERK} 1 / 2$. The relative levels of $\mathrm{pERK} 1 / 2$ in the control cells treated with $10 \%$ FBS and DMSO were $1.169 \pm 0.010$ and $1.087 \pm 0.053$, respectively. The relative pERK $1 / 2$ level was $1.511 \pm 0.044$ in cells treated with Apelin-13 alone. However, the relative pERK1/2 level decreased to $1.1435 \pm 0.041$ in cells 


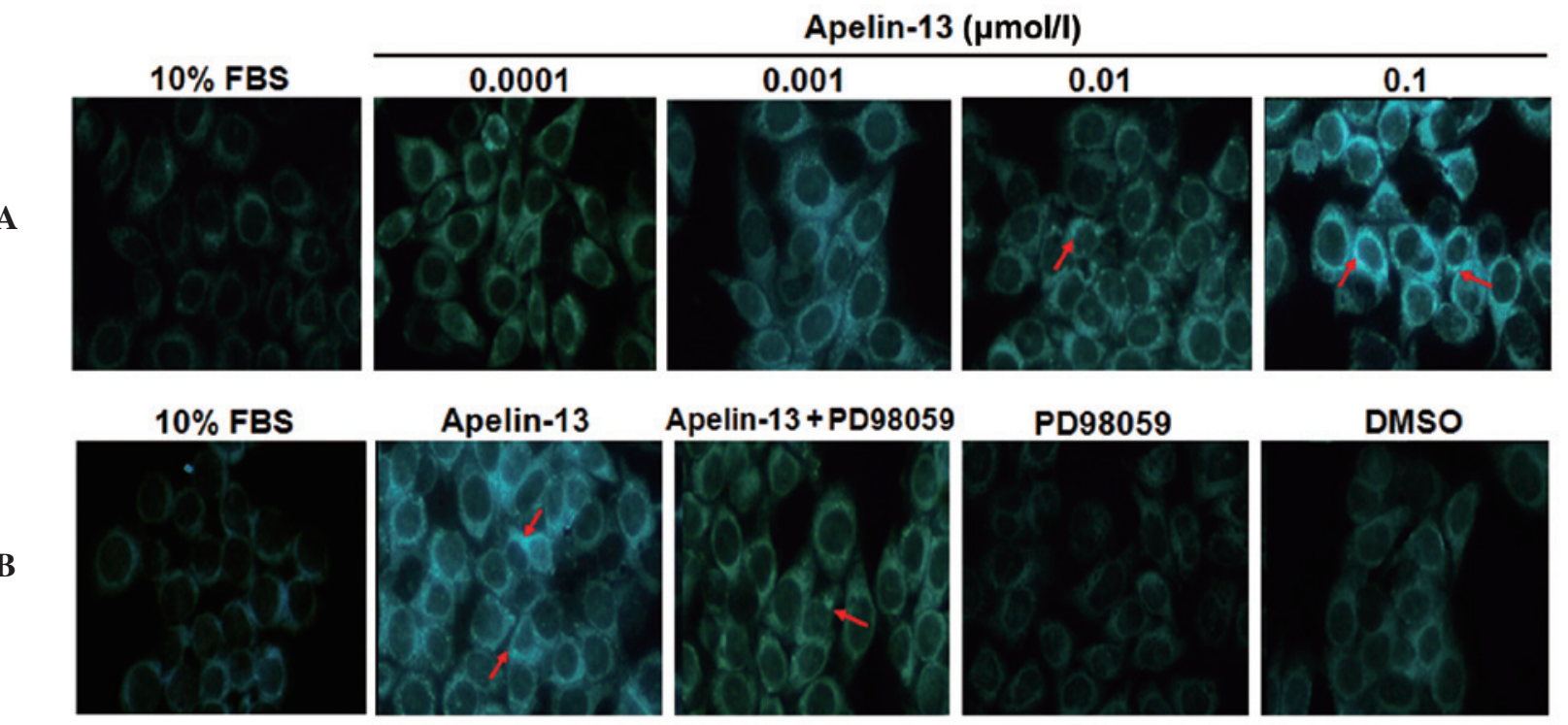

Figure 4. Analysis of autophagy in HepG2 cells subsequent to treatment with Apelin-13 and PD98059. Autophagy of HepG2 cells was observed under inverted fluorescence microscopy following MDC staining. Autophagosomes were stained with blue and green fluorescence. (A) The cells were incubated with 0.0001 , $0.001,0.01$ and $0.1 \mu \mathrm{mol} / 1$ Apelin-13 for $24 \mathrm{~h}$. The cells treated with 10\% FBS were used as a negative control. Representative MDC staining results are shown. Red arrows indicate autophagosomes. (B) Cells were treated with $0.1 \mu \mathrm{mol} / 1$ Apelin-13 alone, $0.1 \mu \mathrm{mol} / 1$ Apelin-13 combined with $10 \mu \mathrm{mol} / 1$ PD98059 or $10 \mu \mathrm{mol} / 1$ PD98059 alone for $24 \mathrm{~h}$. The cells treated with 10\% FBS and DMSO were used as negative controls. Representative MDC staining results are shown. Red arrows indicate autophagosomes. FBS, fetal bovine serum; DMSO, dimethyl sulfoxide; MDC, monodansylcadaverine.

treated with Apelin-13 and PD98059. In cells treated with PD98059 alone, the pERK1/2 relative level was $0.955 \pm 0.023$. Compared with other treatments, the administration of Apelin-13 alone resulted in the highest level of pERK1/2, whereas the administration of PD98059 alone resulted in the lowest level of pERK1/2 $(\mathrm{P}<0.01)$. Therefore, this result reveals that the Apelin-13 inhibitor PD98059 decreased the level of ERK1/2 phosphorylation induced by Apelin-13, further confirming the effect of Apelin-13 on ERK1/2 phosphorylation.

Subsequently, the level of Beclin1 protein and mRNA was detected following treatment with Apelin-13 and PD98059. Consistently, western blot analysis revealed elevated levels of Beclin1 protein in cells treated with Apelin-13 alone, with a relative level of 1.590 \pm 0.010 (Fig. 3C and D). By contrast, the upregulation of Beclin1 expression was blocked in PD98059-treated cells, with a relative level of $0.889 \pm 0.024$. The relative expression of the Beclin1 protein in cells treated with 10\% FBS, Apelin-13 and PD98059, and DMSO were $1.009 \pm 0.045,1.155 \pm 0.039$ and $1.141 \pm 0.018$, respectively. Statistically, there was a significantly increased level of Beclin1 protein in Apelin-13-treated cells and a significantly decreased level of Beclin1 protein in PD98059-treated cells $(\mathrm{P}<0.01)$. In addition, Beclin1 expression at the mRNA level revealed a similar pattern to the expression of the Beclin1 protein, as revealed by RT-PCR (Fig. 3E and F). The relative expression levels of Beclin1 mRNA in cells treated with 10\% FBS, Apelin-13, Apelin-13 and PD98059, PD98059 and DMSO were $1.068 \pm 0.056,1.582 \pm 0.017,1.132 \pm 0.028,0.917 \pm 0.023$ and $1.133 \pm 0.008$, respectively. The differences between Apelin-13-treated, PD98059-treated and control cells were statistically significant $(\mathrm{P}<0.01)$. Thus, these results indicate that the Apelin-13 inhibitor PD98059 inhibited the upregulation of Beclin1 protein and mRNA induced by Apelin-13, further verifying the effect of Apelin-13 on Beclin1 expression.
Apelin-13 promotes and PD98059 inhibits the formation of the autophagosome in HepG2 cells. The aforementioned results indicate that Apelin-13 promotes the formation of the autophagosome in HepG2 cells. In the present study, the autophagy of HepG2 cells following treatment with Apelin-13 and PD98059 was evaluated by MDC staining. Representative results of MDC staining are shown in Fig. 4. The autophagosome was stained with blue and green fluorescence. As shown in Fig. 4A, there was no evident blue or green luminescence in cells treated with $10 \%$ FBS, indicating that no autophagosome was formed. Following treatment with Apelin-13, blue and green fluorescence was evident in the HepG2 cells, indicating that autophagosomes were formed. With the increasing concentration of Apelin-13, more blue and green luminescence autophagosomes were detected in HepG2 cells under inverted fluorescence microscopy. By contrast, in PD98059-treated HepG2 cells, the formation of autophagosomes decreased significantly compared with Apelin-13-treated cells (Fig. 4B). Red arrows indicate representative clusters of fluorescent autophagosomes in the cytoplasm. These data suggest that Apelin-13 induces autophagy in HepG2 cells and that the Apelin-13 inhibitor PD98095 efficiently inhibits the formation of autophagosomes in HepG2 cells treated with Apelin-13.

\section{Discussion}

Hepatocellular carcinoma is a disease that leads to mortality worldwide and is one of the cancers with the highest level of cancer-associated morbidity. This disease is also the third most invasive cancer worldwide (10). In China, hepatocellular carcinoma is the second most invasive cancer (11). Therefore, studies have paid increasing attention to the development of more efficient treatments for this carcinoma. Autophagy is a process through which cells use the lysosome to degrade damaged 
organelles and macromolecules. Autophagy is an important conservative cellular pathway and includes macroautophagy, microautophagy and chaperone-mediated autophagy $(12,13)$. In the present study, the effect of Apelin-13 on HepG 2 cells was observed. Through MDC staining, it was found that treatment with Apelin-13 induced autophagy in HepG2 cells in a dose-dependent manner. In addition, the increase in autophagy was inhibited by the Apelin-13 inhibitor PD98059. This data indicates that Apelin-13 may inhibit hepatocellular carcinoma cells through inducing autophagy and that Apelin-13 may be used to treat hepatocellular carcinoma.

Apelin-13 was also observed to upregulate the expression levels of pERK1/2 and Beclin1 in a dose-dependent manner. In 1999, Beclin1 was observed to be the key gene in the induction of autophagy, and it was the first gene that was identified to inhibit tumors through lysosome degradation (2). Studies have revealed that Beclin1 expression is decreased in cervical cancer (14), ovarian cancer (15) and cerebral tumors (16). Downregulation of Beclin1 expression significantly reduces autophagy in cancer cells $(17,18)$. Therefore, upregulating the expression of Beclin1 may induce autophagy and therefore promote tumor cell death. The present results indicate that activation of the ERK1/2 signaling pathway effectively upregulates the expression of Beclin1 in hepatocellular carcinoma, indicating that the ERK1/2 signaling pathway is one of the mechanisms that mediates the expression of Beclin1. Therefore, the present results indicate that Apenlin-13 may promote autophagy through upregulating Beclin1 and that Apenlin-13 may upregulate Beclin1 expression through the ERK1/2 signaling pathway. Subsequent to treatment with the Apelin-13 inhibitor PD98059, the level of autophagy and pERK1/2 and Beclin1 expression were decreased. This further verified the promoting effect of Apelin-13 on autophagy and Beclin1 expression. However, whether Apelin-13 regulates autophagy and Beclin1 expression through other pathways requires additional investigation.

In summary, the present study found that Apelin-13 induced autophagy in hepatoma cells through ERK1/2 signaling pathway-dependent upregulation of Beclin1. Fundamental low-level autophagy activity is vital to maintain a steady state in normal and tumor cells. Excessive autophagy may lead to the death of cells (19). Additionally, autophagy plays dual roles in tumor development (20). As a regulatory protein of autophagy, Beclin1 efficiently inhibits the development of tumors by inhibiting angiogenesis (21). Therefore, the present results provide experimental evidence for the use of Apelin-13 in the treatment of hepatocellular carcinoma.

\section{Acknowledgements}

This study was supported by grants from the National Natural Science Foundation of China (grant nos. 81102230 and 81270420).

\section{References}

1. Meijer AJ and Codogno P: Regulation and role of autophagy in mammalian cells. Int J Biochem Cell Biol 36: 2445-2462, 2004.
2. Liang XH, Jackson S, Seaman M, Brown K, Kempkes B, Hibshoosh $\mathrm{H}$ and Levine B: Induction of autophagy and inhibition of tumorigenesis by beclin 1. Nature 402: 672-676, 1999.

3. Shoji-Kawata S, Sumpter R, Leveno M, Campbell GR, Zou Z, Kinch L, Wilkins AD, Sun Q, Pallauf K, MacDuff D, et al: Identification of a candidate therapeutic autophagy-inducing peptide. Nature 494: 201-206, 2013.

4. Furuya N, Yu J, Byfield M, Pattingre S and Levine B: The evolutionarily conserved domain of Beclin 1 is required for Vps34 binding, autophagy and tumor suppressor function. Autophagy 1: 46-52, 2005.

5. Pattingre $\mathrm{S}$, Bauvy $\mathrm{C}$ and Codogno P: Amino acids interfere with the ERK1/2-dependent control of macroautophagy by controlling the activation of Raf-1 in human colon cancer HT-29 cells. J Biol Chem 278: 16667-16674, 2003.

6. Huang YC, Hung WC, Chen WT, Yu HS and Chai CY: Sodium arsenite-induced DAPK promoterhypermethylation and autophagy via ERK1/2 phosphorylation in humanuroepithelial cells. Chem Biol Interact 181: 254-262, 2009.

7. Tatemoto K, Hosoya M, Habata Y, Fujii R, Kakegawa T, Zou MX, Kawamata Y, Fukusumi S, Hinuma S, Kitada C, et al: Isolation and characterization of a novel endogenous peptide ligand for the human APJ receptor. Biochem Biophys Res Commun 251: 471-476, 1998.

8. Li F, Li L, Qin X, Pan W, Feng F, Chen F, Zhu B, Liao D, Tanowitz H, Albanese C, et al: Apelin-induced vascular smooth muscle cell proliferation: The regulation of cyclin D1. Front Biosci 13: 3786-3792, 2008.

9. Biederbick A,Kern HF and Elsässer HP: Monodansylcadaverine (MDC) is a specific in vivo marker for autophagic vacuoles. Eur J Cell Biol 66: 3-14, 1995.

10. D'Alessandro LA, Meyer R and Klingmüller U: Hepatocellular carcinoma: A systems biology perspective. Front Physiol 4: 28, 2013.

11. He J, Gu D, Wu X, Reynolds K, Duan X, Yao C, Wang J, Chen CS, Chen J, Wildman RP, et al: Major causes of death among men and women in China. N Engl J Med 353: 1124-1134, 2005.

12. Klionsky DJ: The molecular machinery of autophagy: Unanswered questions. J Cell Sci 118: 7-18, 2005.

13. Massey AC, Zhang C and Cuervo AM: Chaperone-mediated autophagy in aging and disease. Curr Top Dev Biol 73: 205-235, 2006.

14. Wang ZH, Xu L, Duan ZL, Zeng LQ, Yan NH and Peng ZL: Beclin 1 mediated macroautophagy involves regulation of caspase-9 expression in cervical cancer HeLa cells. Gynecol Oncol 107: 107-113, 2007.

15. Duan ZL, Peng ZL and Wang ZH: Expression and involved sign alransduction pathway of autophagy gene Beclin 1 in epithelial ovarian cancer. Sichuan Da Xue Xue Bao Yi Xue Ban 38: 239-242, 2007 (In Chinese).

16. Miracco C, Cosci E, Oliveri G, Luzi P, Pacenti L, Monciatti I, Mannucci S, De Nisi MC, Toscano M, Malagnino V, et al: Protein and mRNA expression of autophagy gene Beclin 1 in human brain tumours. Int J Oncol 30: 429-436, 2007.

17. Roesly HB, Khan MR, Chen HD, Hill KA, Narendran N, Watts GS, Chen X and Dvorak K: The decreased expression of Beclin-1 correlates with progression to esophageal adenocarcinoma: The role of deoxycholic acid. Am J Physiol Gastrointest Liver Physiol 302: G864-G872, 2012.

18. Zhou WH, Tang F, Xu J, Wu X, Yang SB, Feng ZY, Ding YG, Wan XB, Guan Z, Li HG, et al: Low expression of Beclin 1, associated with high Bcl-XL, predicts a malignant phenotype and poor prognosis of gastric cancer. Autophagy 8: 389-400, 2012.

19. Yang $Z$ and Klionsky DJ: Eaten alive: A history of macroautophagy. Nat Cell Biol 12: 814-822, 2010.

20. Apel A, Zentgraf H, Büchler MW and Herr I: Autophagy - a double-edged sword in oncology. Int J Cancer 125: 991-995, 2009.

21. Lee SJ, Kim HP, Jin Y, Choi AM and Ryter SW: Beclin 1 deficiency is associated with increased hypoxia-induced angiogenesis. Autophagy 7: 829-839, 2011. 